\title{
Analysis of Cumulant Moments in High Energy Hadron-Hadron Collisions by Truncated Multiplicity Distributions
}

\author{
Noriaki Nakajima, Minoru Biyajima, and Naomichi Suzuki ${ }^{1}$ \\ Department of Physics, Shinshu University, Matsumoto 390, Japan \\ ${ }^{1}$ Matsusho Gakuen Junior College, Matsumoto 390-12, Japan
}

\begin{abstract}
Oscillatory behavior of cumulant moments obtained from the experimental data in $p p$ collisions and $\bar{p} p$ collisions are analyzed by the modified negative binomial distribution (MNBD) and the negative binomial distribution (NBD). Both distributions well describe the cumulant moments obtained from the data. This fact shows sharp contrast to the result in $e^{+} e^{-}$collisions, which is described by the the MNBD much better than by the NBD.
\end{abstract}

\section{Introduction}

Recently a prediction is made by the QCD calculations that the cumulant moment of multiplicity distribution possesses an oscillatorye behavior [1]]. Furthermore, analysis of the cumulant moments in hadron-hadron $h h$ and $e^{+} e^{-}$collisions shows that the $j$-th order normalized cumulant moment of observed charged multiplicity distributions oscillates irregularly around the zero with increasing the rank $j$ [2]. At present calculated results by the QCD explain the behavior of the data only qualitatively.

It is well known that the cumulant moment of negative binomial distribution (NBD) calculated from the multiplicity generating function is positive and decreases monotonously as the rank increases. However, it is shown in Ref. [3] that oscillatory behavior of cumulant moment appears if it is calculated from the truncated NBD. The possibility is pointed out that the cumulant moments of the modified negative binomial distribution (MNBD) calculated from the generating function change sign alternatively as the rank of the cumulant moments increases [4]. Cumulant moments of negatively charged particles and charged particles obtained from the data in $e^{+} e^{-}$collisions have been analyzed by the MNBD [4]. The result shows that the cumulant moments of the negatively charged particles obtained from the data show rather regular oscillation around zero; those with evenh rank are smaller than two adjacent moments at least up to the 6th rank. The 
cumulant moments of the negatively charged and charged particles obtained from the data are well described by the MNBD, if it is truncated at the maximum of the observed negatively charged multiplicity. However, in $e^{+} e^{-}$collisions, oscillation of cumulant moments calculated from the truncated NBD is very weak comparing with that obtained from the data.

In this paper, we analyze the cumulant moments of charged particles observed in $p p$ and $\bar{p} p$ collisions, by the MNBD and the NBD.

The j-th order normalized cumulant $K_{j}$ of charged particles is expressed by the normalized factorial moments $F_{l} \quad(l=1,2, \cdots)$ of charged particles as,

$$
\begin{aligned}
& K_{1}=F_{1}, \\
& K_{j}=F_{j}+\sum_{m=1}^{j-1}{ }_{j-1} C_{m-1} F_{j-m} K_{m}, \quad j=2,3, \cdots,
\end{aligned}
$$

where

$$
F_{j}=<n_{\mathrm{ch}}\left(n_{\mathrm{ch}}-1\right) \cdots\left(n_{\mathrm{ch}}-j+1\right)>/<n_{\mathrm{ch}}>^{j}
$$

The $H_{j}$ moment is defined by

$$
H_{j}=K_{j} / F_{j}
$$

\section{A Stochastic Process}

In [5], a birth process with an immigration is taken forc a model of particle productions,

$$
\begin{gathered}
\frac{\partial P(n ; t)}{\partial t}=-\lambda_{0} P(n ; t)+\lambda_{0} P(n-1 ; t) \\
-\lambda_{2} n P(n ; t)+\lambda_{2}(n-1) P(n-1 ; t),
\end{gathered}
$$

where $\lambda_{0}$ denotes an immigration rate, and $\lambda_{2}$ a birth rate. An initial condition of Eq.(3) is taken as a binomial distribution,

$$
P(n ; t=0)={ }_{N} C_{n} \alpha^{n} \beta^{N-n}, \quad \beta=1-\alpha .
$$

The generating function $\Pi(z)$ of the multiplicity distribution $P(n)$ is defined by

$$
\Pi(z)=\sum_{n=0} P(n) z^{n}
$$

Then, we have the generating function for the distribution $P(n ; t=T)$,

$$
\Pi(z ; t=T)=\frac{1}{[1-p(z-1)]^{k}}\left(\frac{1-[p-\alpha(1+p)](z-1)}{1-p(z-1)}\right)^{N}
$$


where

$$
p=\exp \left[\lambda_{2} T\right]-1, \quad k=\lambda_{0} / \lambda_{2}
$$

If the branching process is governed by neutral particles, and those particles finally decay into charged pairs with a probability $a$ or neutral particles with $(1-a)$. Then, from Eq.(6), we havey the generating function for charged particles,

$$
G(w)=\Pi\left(a\left(w^{2}-1\right)+1 ; t=T\right),
$$

where the variable $z$ in Eq.(6) is replaced by $a w^{2}+(1-a)$. The generating function $\Pi(z)$ for negatively charged particles is therefore written as

$$
\begin{aligned}
\Pi(z) & =\left[1-r_{1}(z-1)\right]^{N}\left[1-r_{2}(z-1)\right]^{-N-k} \\
r_{1} & =a(p-\alpha(1+p)), \quad r_{2}=a p
\end{aligned}
$$

It should be noted that in Eq.(7), $N$ is a positive integer, $r_{1}$ is real $\left(r_{1}<0\right.$ or $\left.r_{1} \geq 0\right)$, and $r_{2}>0$.

The probability distribution is obtained from $\Pi(z)$ as

$$
\begin{aligned}
P(0) & =\Pi(0)=\frac{\left(1+r_{1}\right)^{N}}{\left(1+r_{2}\right)^{N+k}} \\
P(n) & =\left.\frac{1}{n !} \frac{\partial^{n} \Pi(z)}{\partial z^{n}}\right|_{z=0} \\
& =\frac{1}{n !}\left(\frac{r_{1}}{r_{2}}\right)^{N} \sum_{j=0}^{N}{ }_{N} C_{j} \frac{\Gamma(k+n+j)}{\Gamma(k+j)}\left(\frac{r_{2}-r_{1}}{r_{1}}\right)^{j} \frac{r_{2}^{n}}{\left(1+r_{2}\right)^{n+k+j}}, \quad n=1,2, \cdots
\end{aligned}
$$

If $k=0$, the summation on the right hand side of Eq.(8) runs from $j=1$ up to $j=N$, and it is called the MNBD [5], [6]. The second paper in [6] corresponds to the case $\alpha=1$, which leads to $r_{1}<0$. If $N=0$, Eq.(8) a is reduced to the NBD.

If $k=0$, parameters $r_{1}$ and $r_{2}$ are expressed by the average multiplicity $\langle n\rangle$ and $C_{2}$ moment of negatively charged particles respectively as,

$$
\begin{aligned}
& r_{1}=\frac{1}{2}\left(C_{2}-1-\frac{1}{\langle n\rangle}-\frac{1}{N}\right)\langle n\rangle, \\
& r_{2}=\frac{1}{2}\left(C_{2}-1-\frac{1}{\langle n\rangle}+\frac{1}{N}\right)\langle n\rangle .
\end{aligned}
$$

If $N=0$, parameter $r_{1}$ is not contained in Eq.(8), and $r_{2}$ is given by

$$
r_{2}=\frac{<n>}{k}
$$




\section{Analysis of the experimental data}

The cumulant moments of charged particles at the ISR in $p p$ collisions [7], and of the UA5 collaboration in $\bar{p} p n$ collisions [8] are analyzed by the MNBD and NBD in this paper. Equation (8) is applied to the multiplicity distribution of negatively charged particles. The parameters used in the analysis are shown in Table I. Those are determined by the minimum chi-square $\left(\chi_{\min }^{2}\right)$ fit to the observed multiplicity distributionsm of negatively charged particles. As is seen from the Table I, the $\chi_{\min }^{2}$ values of the MNBD fit are smaller than those of the NBD fit in both energy regions.

Factorial moments of charged particles are calculated as

$$
\begin{aligned}
f_{j}^{\mathrm{ch}} & =<n_{\mathrm{ch}}\left(n_{\mathrm{ch}}-1\right) \cdots\left(n_{\mathrm{ch}}-j+1\right)> \\
& =\sum_{n}^{n_{\max }}\left(2 n+n_{0}\right)\left(2 n+n_{0}-1\right) \cdots\left(2 n+n_{0}-j+1\right) P(n), \quad j=1,2, \cdots,
\end{aligned}
$$

where $P(n)$ is given by Eq.(8). In Eq.(9), $n_{\max }$ denotes the maximum of the observed negatively charged multiplicity, and $n_{0}$ is taken as $n_{0}=2$ for $p p$ collisions, and $n_{0}=0$ for $\bar{p} p$ collisions.

Then, cumulant moments of charged particles are calculated from Eqs. (1), (2) and (9) with the parameters shown in Table I. Those are compared with the cumulant moments of charged particles at the ISR in Figs.1a, b, c and d, and those for the UA5 Collaboration from $200 \mathrm{GeV}$ to $900 \mathrm{GeV}$ in Figs.2a, b and c. Both the MNBD and the NBD well describe the behavior of the data.

\section{Concluding remarks}

The cumulant moments of observed multiplicity distributions of charged particles in $p p$ and $\bar{p} p$ collisions are analyzed by the MNBD and the NBD. The cumulant moments obtained from the data oscillate rather irregularly as the rank of the moments increases. Those behavior are well described by the calculated results by the MNBD as well as by the NBD. The results in $p p$ and $\bar{p} p$ collisions are much different from those in $e^{+} e^{-}$ collisions, where the data is described much better by the MNBD than by the NBD.

The cumulant moments of negatively charged particles obtained from the data in $p p$ or $\bar{p} p$ collisions does not show the regular oscillations contrary to those in $e^{+} e^{-}$collisions.

The characteristics of the cumulant moments in $h h$ and $e^{+} e^{-}$collisions, discussed above, are reduced mainly to the difference ofd the hadronization between the two processes.

\section{Acknowledgements}

One of the authors M. B. is partially supported by the Grant-in Aid for Scientific Research from the Ministry of Education, Science and Culture (No. 06640383). N. S. thanks for the financial support by Matsusho Gakuen Junior College. 


\section{References}

[1] I. M. Dremin and V. A. Nechitailo, JETP Lett. 58(1993)881,

I. M. Dremin and R. Hwa, Phys. Rev. D49, 5805a (1994)

I. M. Dremin, Phys. Lett. B341, 95 (1994)

[2] I. M. Dremin et al., Phys. Lett. B336, 119 (1994)

[3] R. Ugoccini, A. Giovannini and S. Lupia, Phys. Lett.i B342, 387 (1995)

[4] N. Suzuki, M. Biyajima and N. Nakajima, Phys. Rev. D53, 3582 (1996); New analysis of Cumulant Moments in $e^{+} e^{-}$Collisions by SLD Collaboration by Truncated Multiplicity Distributions, submitted to Phys. Rev D.

[5] N. Suzuki, M. Biyajima and G. Wilk, Phys. Lett. B268, 447 (1991)

[6] P. V. Chliapnikov and O. G. Tchikilev, Phys. Lett. B242, 275 (1990);

Phys. Lett. B282, 471 (1992)

P. V. Chliapnikov and O. G. Tchikilev and V. A. Uvarov, Phys. Lett. B352, 461 (1995)

[7] A. Breakstone et al., Phys. Rev. D30, 528( 1984)

[8] UA5 Collaboration, G. J. Alner, Phys. Rep. 154, 247 (1987);

UA5 Collaboration, R. E. Ansorge et al., Z. Phys. C43, 357 (1989) 


\section{Table caption}

Table 1 The parameters of the MNBD and the NBD used in the analysis of the cumulant moments in $p p$ collisions, and in $\bar{p} p$ collisions.

\section{Figure captions}

Fig. 1 The normalized cumulant moments of charged particles in $p p$ collisions. The full circles are obtained from the data [0] at a) $\sqrt{s}=30.4 \mathrm{GeV}$, b) $\sqrt{s}=44.5 \mathrm{GeV}$, c) $\sqrt{s}=52.6 \mathrm{GeV}$, and d) $\sqrt{s}=62.2 \mathrm{GeV}$. The solid line is obtained from our calculations with the MNBD, and the dashed line is with the NBD.

Fig. 2 The normalized cumulant moments of charged particles in $\bar{p} p$ collisions. The full circles are obtained from the data [8] from a) $\sqrt{s}=200 \mathrm{GeV}$, b) $\sqrt{s}=546 \mathrm{GeV}$, and c) $\sqrt{s}=900 \mathrm{GeV}$. The dashed lines are obtained from our calculations. 


\begin{tabular}{cccccc}
\hline MNBD & $\sqrt{s}[\mathrm{GeV}]$ & $\mathrm{N}$ & $\langle n\rangle$ & $C_{2}$ & $\chi^{2} / \mathrm{NDF}$ \\
\hline & 30.4 & 6 & $4.214 \pm 0.062$ & $1.2993 \pm 0.0034$ & $11.0 / 11$ \\
& 44.5 & 6 & $5.064 \pm 0.053$ & $1.2775 \pm 0.0052$ & $6.8 / 16$ \\
& 52.6 & 7 & $5.373 \pm 0.049$ & $1.2880 \pm 0.0067$ & $5.0 / 18$ \\
& 62.2 & 12 & $5.821 \pm 0.061$ & $1.2660 \pm 0.0077$ & $23.3 / 17$ \\
& 200 & 4 & $10.69 \pm 0.13$ & $1.2633 \pm 0.0113$ & $7.7 / 28$ \\
& 546 & 4 & $14.65 \pm 0.09$ & $1.2746 \pm 0.0040$ & $67.0 / 44$ \\
& 900 & 3 & $17.90 \pm 0.18$ & $1.2955 \pm 0.0082$ & $57.7 / 52$ \\
\hline \hline NBD & $\sqrt{s}[\mathrm{GeV}]$ & $\langle n\rangle$ & $k$ & $\chi^{2} / \mathrm{NDF}$ \\
\hline & 30.4 & & $4.370 \pm 0.059$ & $21.44 \pm 3.76$ & $27.5 / 12$ \\
& 44.5 & & $5.086 \pm 0.051$ & $13.73 \pm 1.51$ & $14.7 / 17$ \\
& 52.6 & & $5.384 \pm 0.049$ & $9.98 \pm 0.65$ & $5.5 / 19$ \\
& 62.2 & & $5.823 \pm 0.062$ & $10.77 \pm 0.79$ & $23.9 / 18$ \\
& 200 & & $10.56 \pm 0.13$ & $5.66 \pm 0.36$ & $4.3 / 29$ \\
& 546 & & $14.65 \pm 0.09$ & $4.82 \pm 0.10$ & $89.6 / 45$ \\
& 900 & & $16.81 \pm 0.18$ & $3.47 \pm 0.12$ & $73.7 / 53$ \\
\hline
\end{tabular}

Table 1 


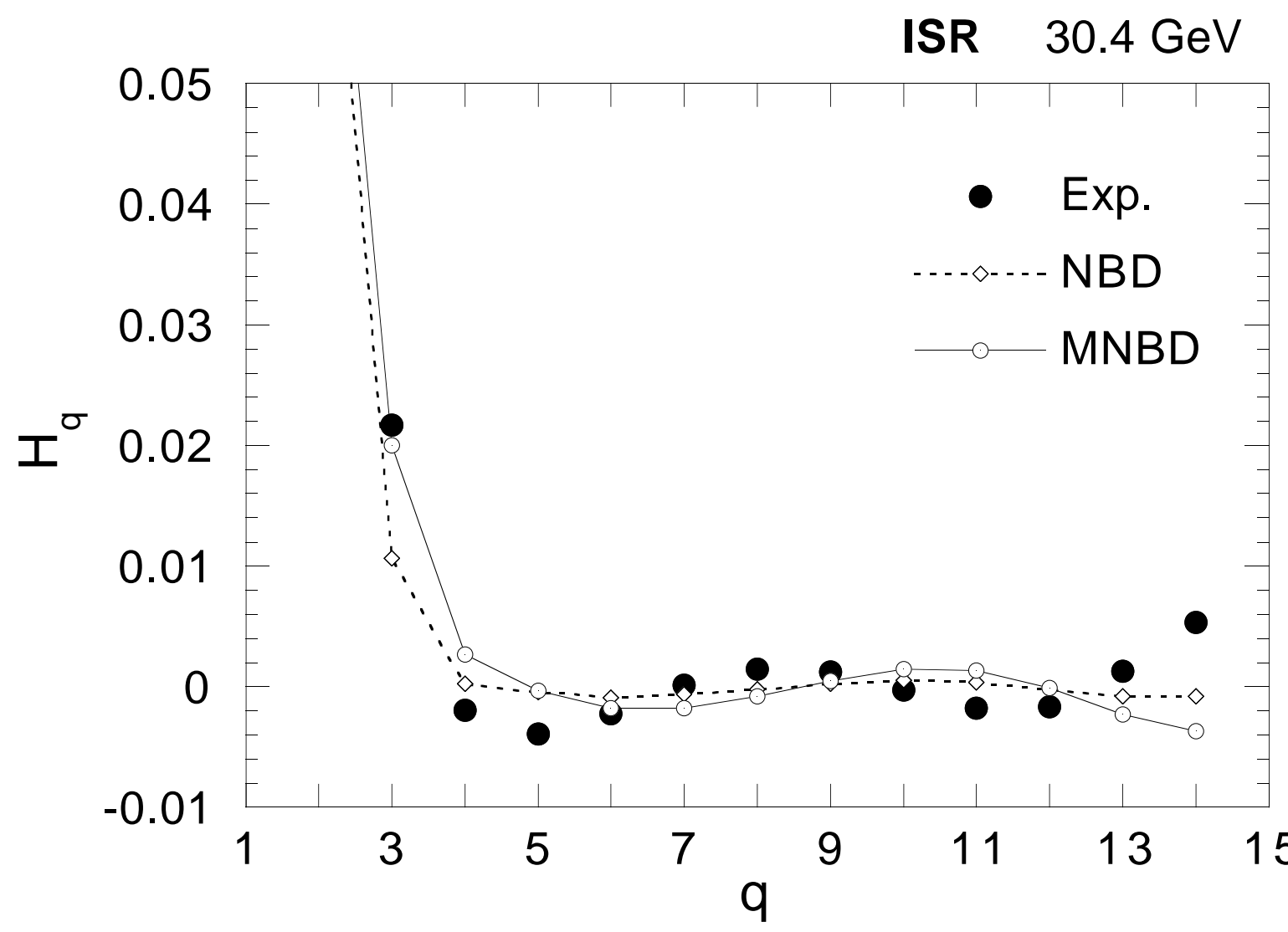

Fig.1a 


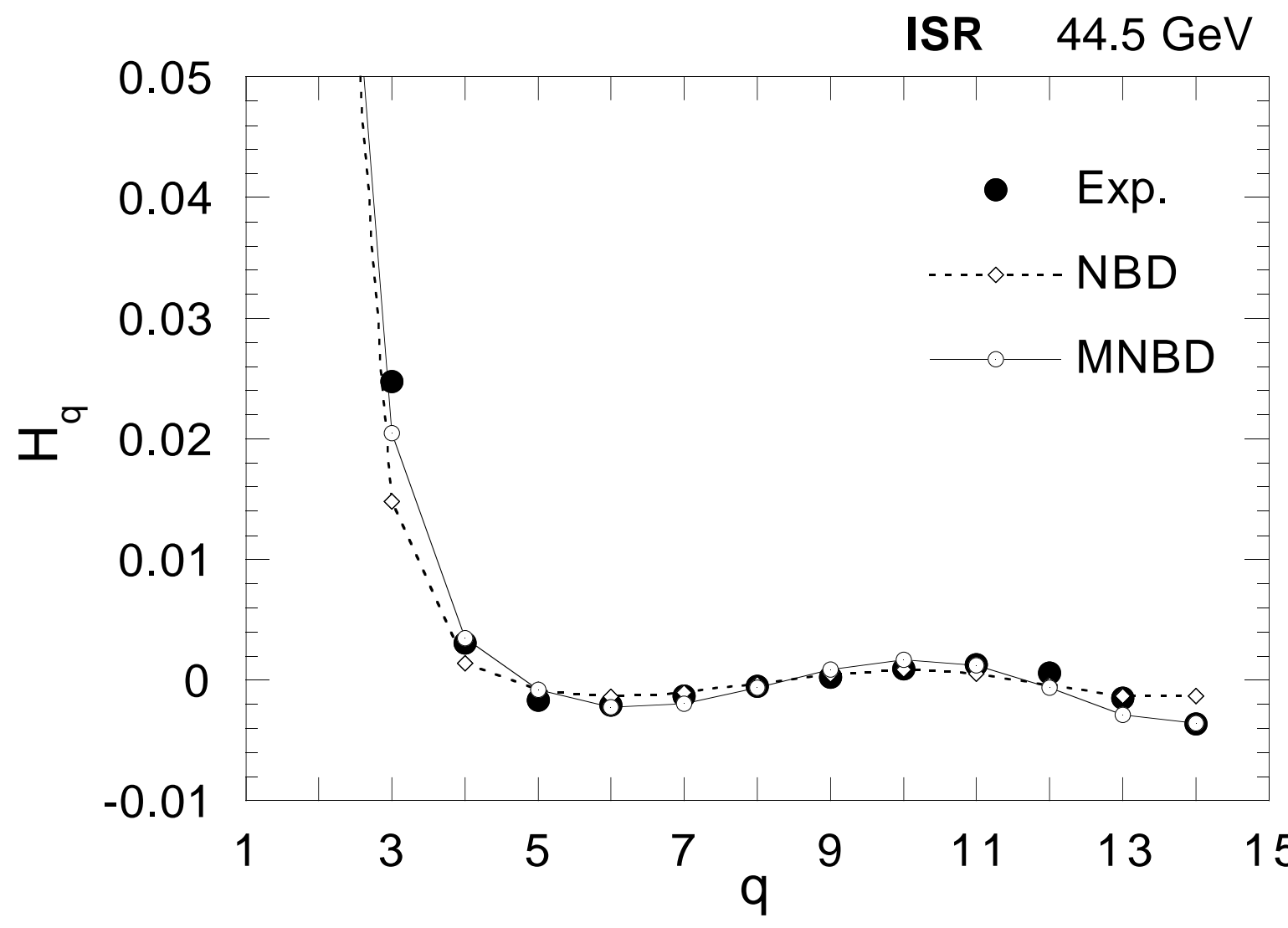

Fig. 1b 


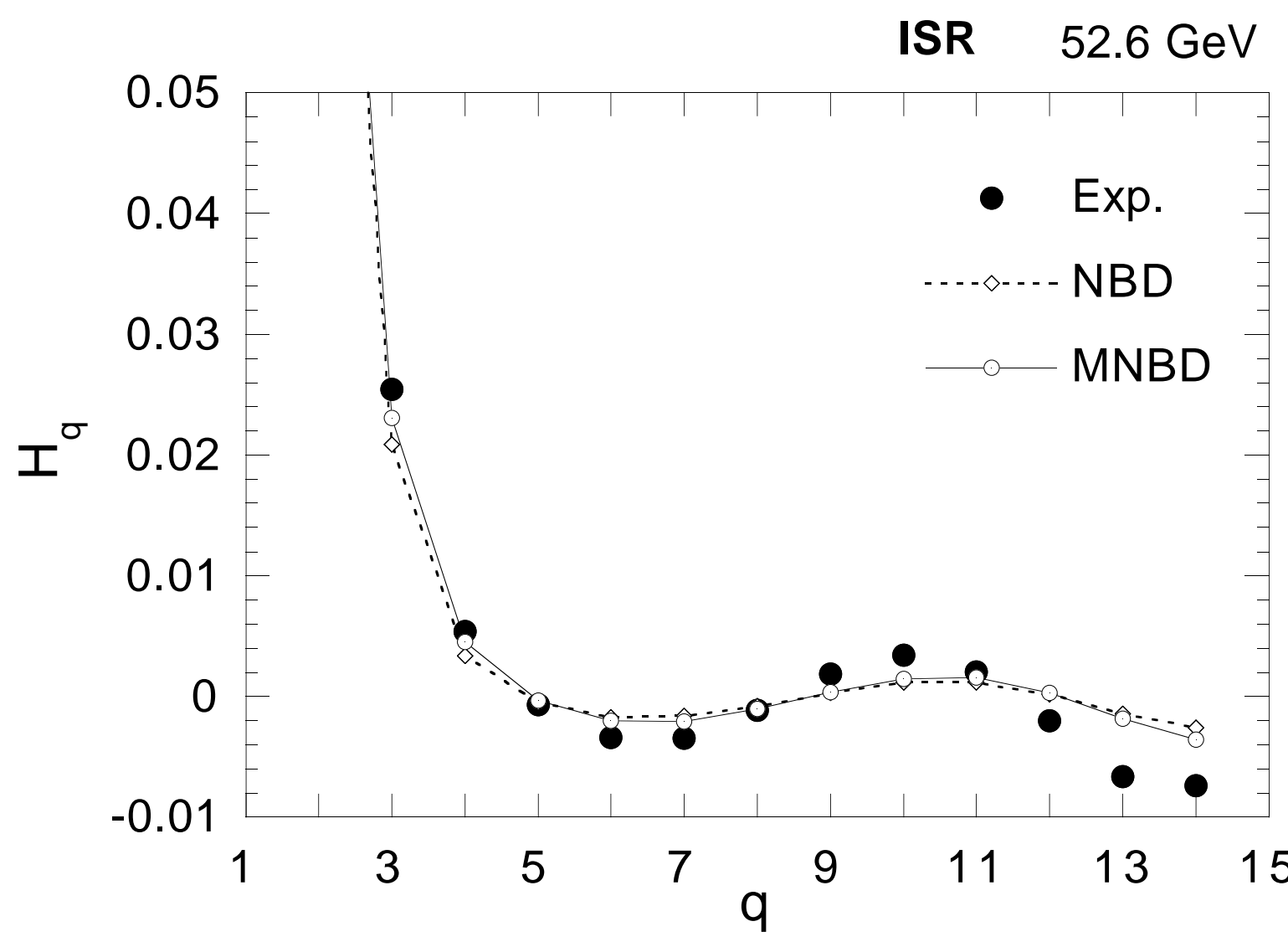

Fig.1c 


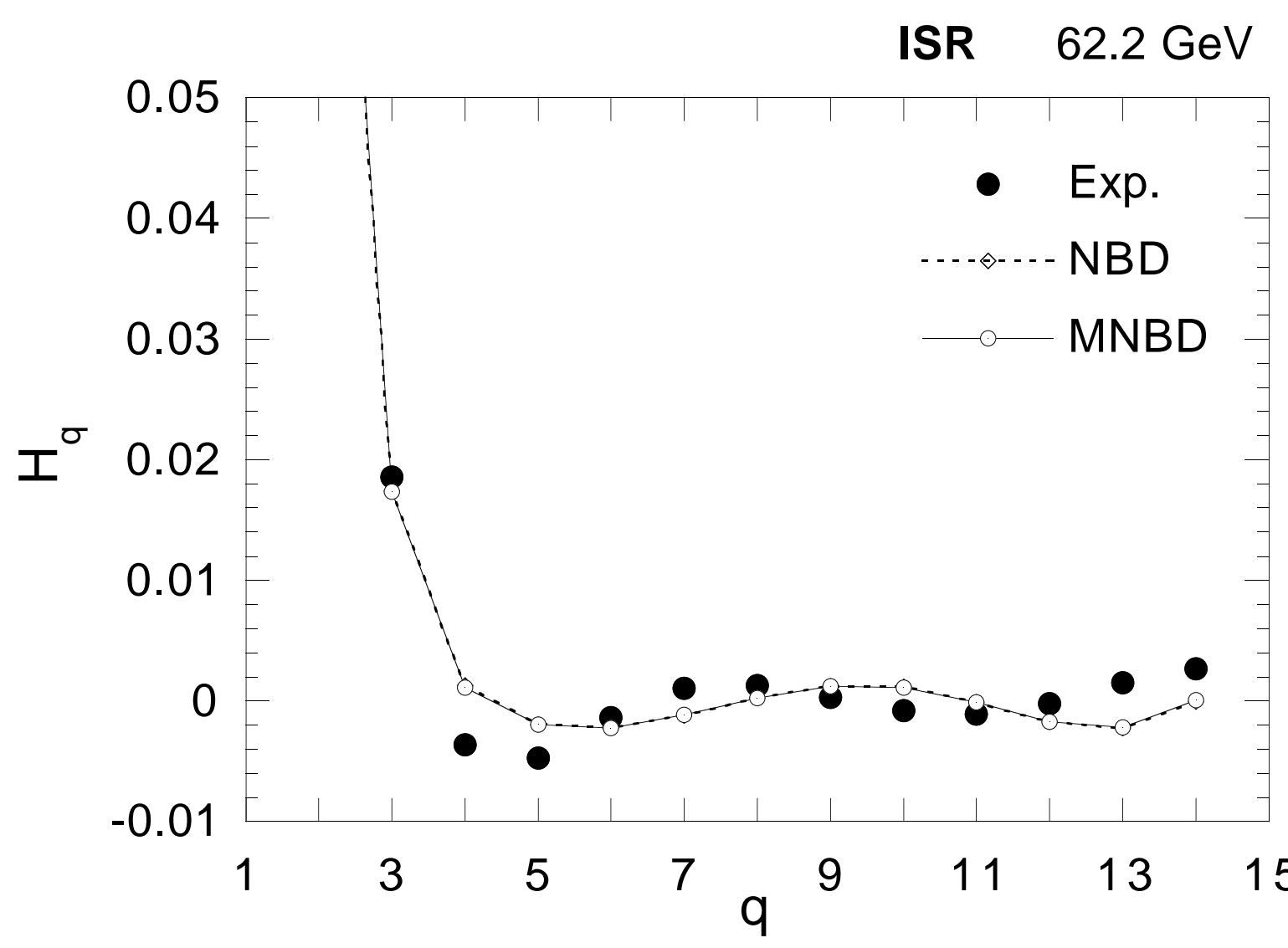

Fig.1d 


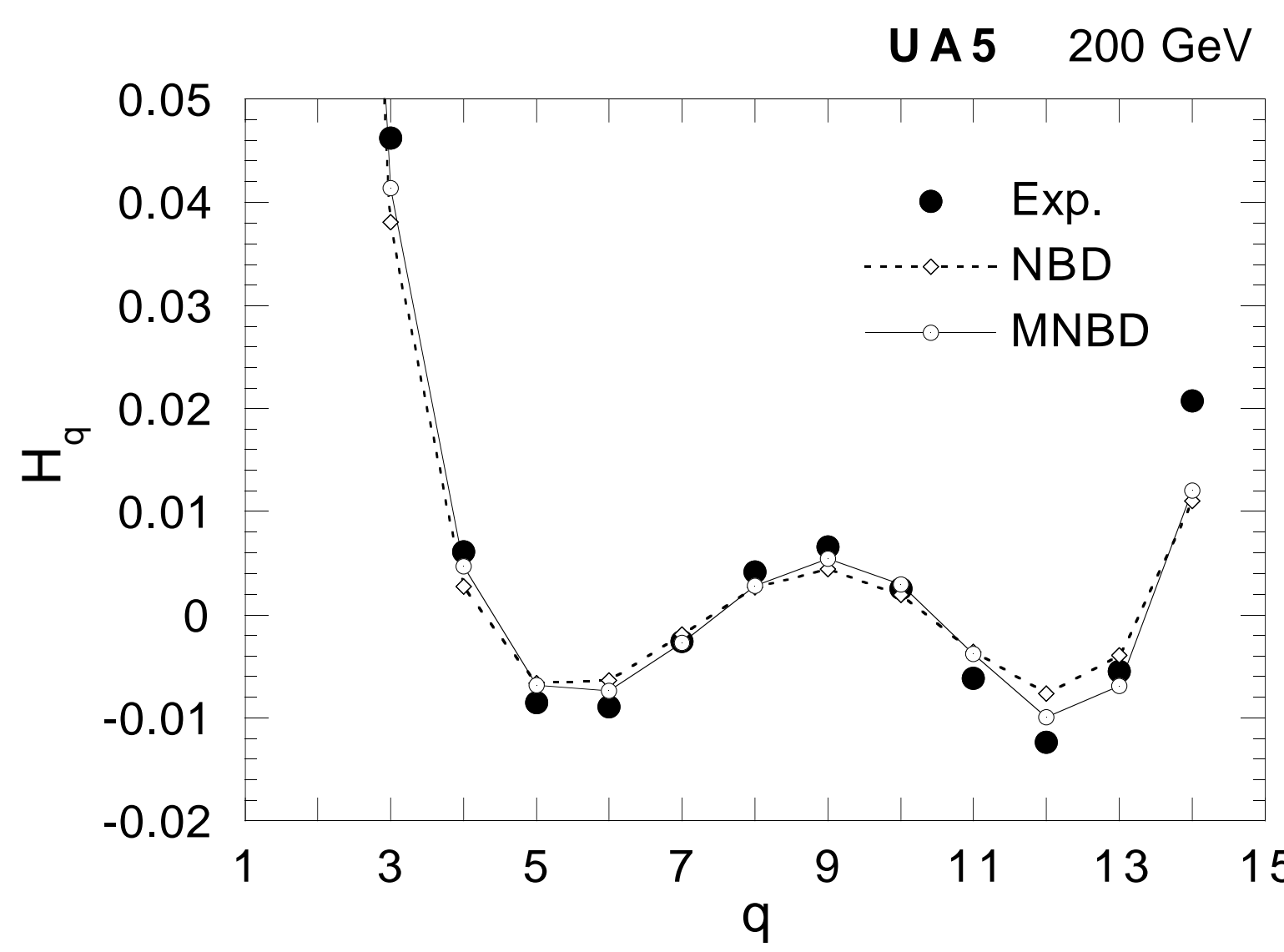

Fig. 2a 


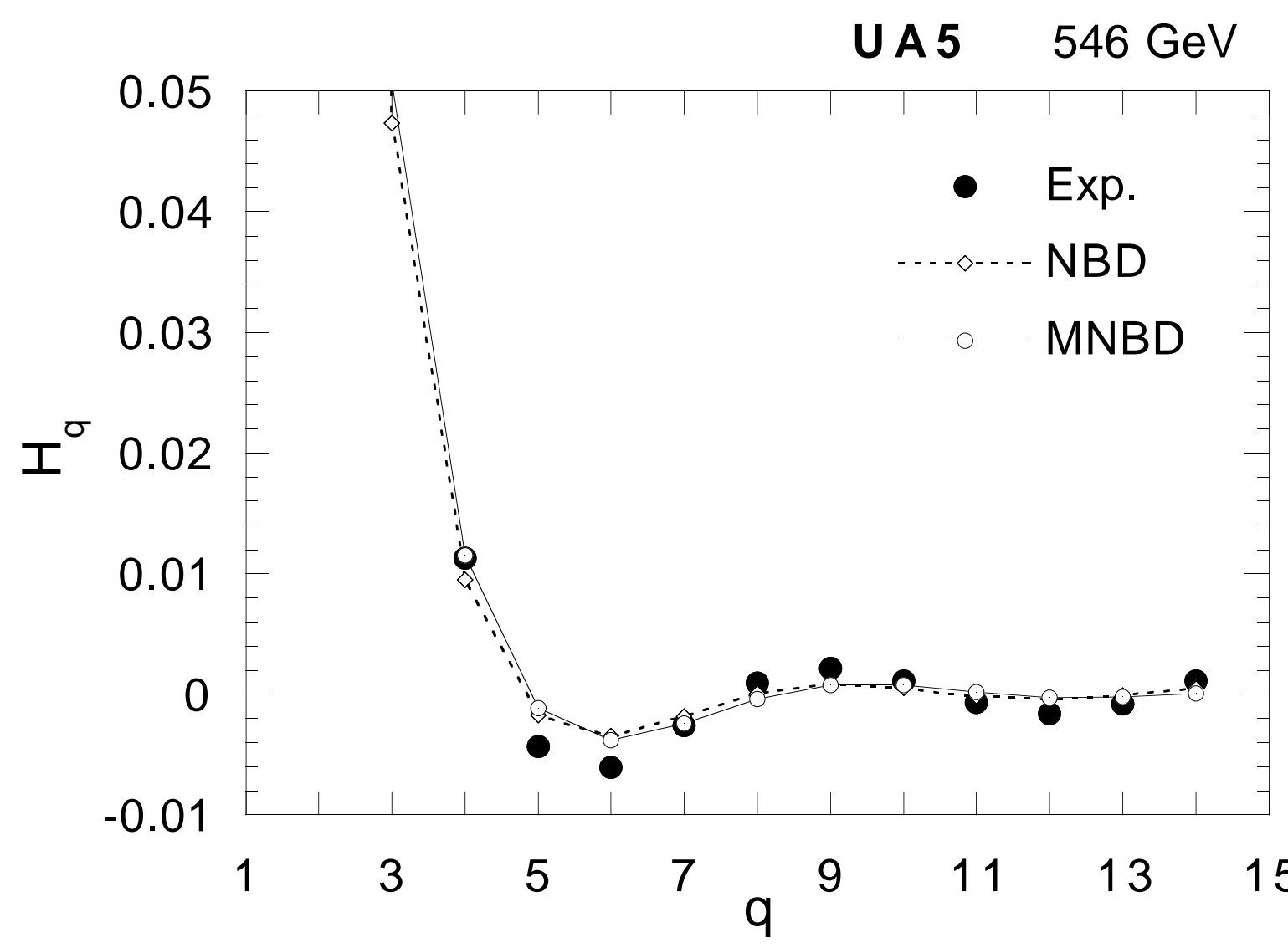

Fig. 2b 


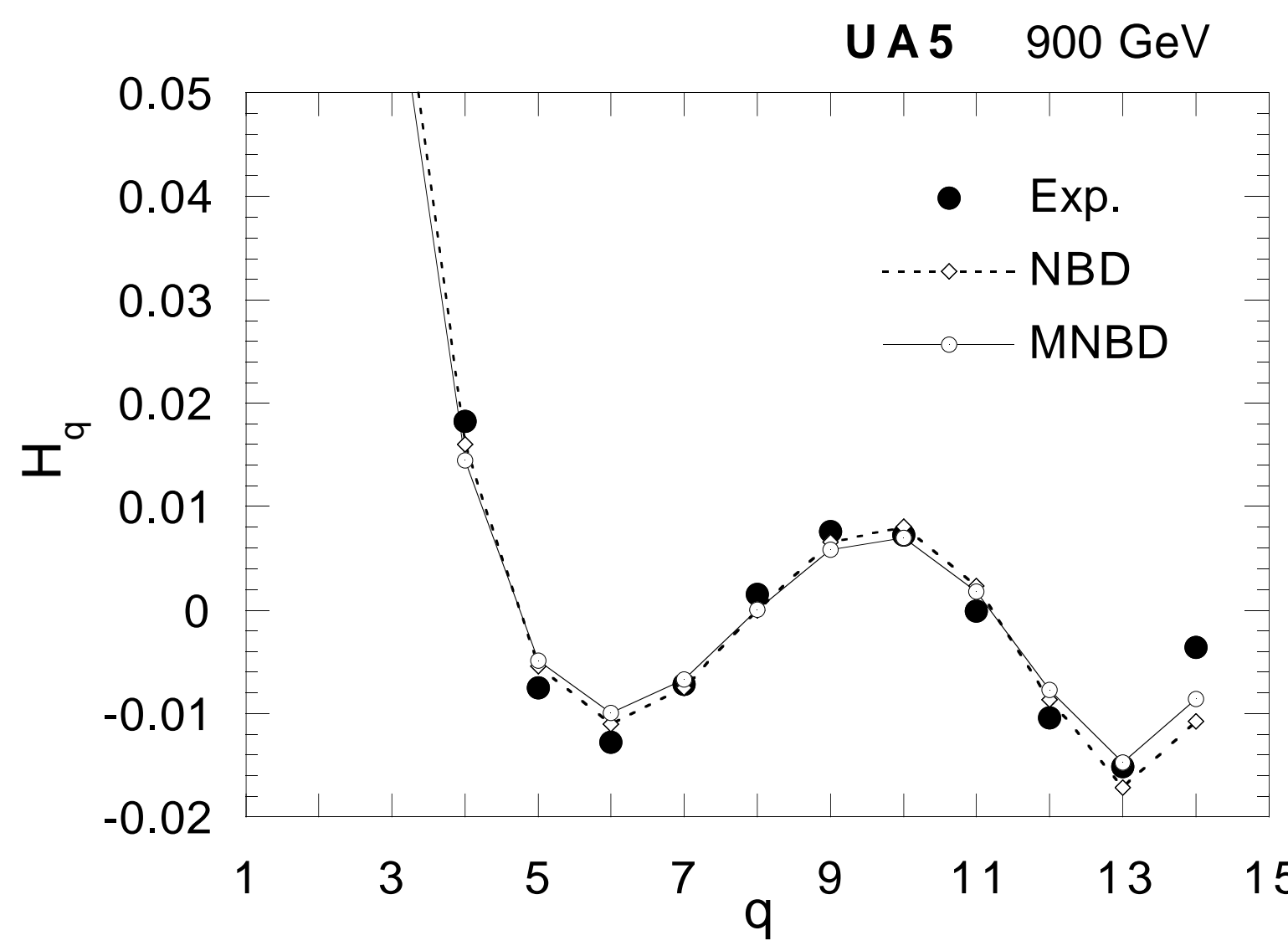

Fig.2c 